\title{
Awake craniotomy for clipping of unruptured aneurysm: anesthesia concerns, management and challenges
}

\author{
Wan Mohd Nazaruddin Wan Hassan, MD, MMed (Anesth) ${ }^{1}$,Sanihah Che Omar,MBBS, MMed \\ (Anesth) ${ }^{2}$, Wong Teck Fui, MBBS 3 Zamzuri Idris, MBBS, MMed (Neurosurgery) ${ }^{4}$, Regunath \\ Kandasamy, MBBS, MMed (Neurosurgery) ${ }^{5}$
}

1. Associate Professor, 2. Senior Lecturer, 3. Postgraduate Candidate, Department of Anesthesiology and Intensive Care, 4. Professor, 5. Senior Lecturer, Department of Neurosciences

1-5. School of Medical Sciences \& Hospital USM, Universiti Sains Malaysia, Kota Bharu, Kelantan, Malaysia.

Correspondence: Associate Professor Dr. Wan Mohd Nazaruddin Wan Hassan, Department of Anesthesiology and Intensive Care, School of Medical Sciences, Universiti Sains Malaysia, 16150 Kota Bharu, Kelantan, (Malaysia); Tel: +60199630385; Fax: +6097653370; E-mail: drnaza anaest@yahoo.co.uk

\section{Abstract}

Awake craniotomy is a neurosurgical technique performed either as an asleep-awake-asleep technique or a monitored anesthesia care technique, in combination with a scalp block. The surgery indication has been well documented for brain tumor excision at the eloquent cortex, epilepsy and deep brain stimulation surgery. However, this technique's use during unruptured cerebral aneurysm clipping has only been recently reported. The main goal of awake craniotomy for unruptured cerebral aneurysm clipping is to prevent the risk of ischemia during temporary and permanent clipping by immediate awake neurological assessment during surgery. There have been limited reports on this surgery's anesthesia issues. The aim of our case report is to discuss the anesthesia concerns, management and challenges during our first successful experience of this surgery.

Key words: Awake craniotomy; Clipping; Cerebral aneurysm; Scalp block

Citation: Hassan WMNW, Omar SC, Fui WT, Idris Z, Kandasamy R. Awake craniotomy for clipping of unruptured aneurysm: anesthesia concerns, management and challenges. Anaesth. pain intensive care 2021;25(1):97-103. DOI: $10.35975 / a p i c . v 25 i 1.1436$

Received: 2 July 2020, Reviewed: 10 August 2020, Accepted: 3 September 2020

\section{Introduction}

Awake craniotomy has recently been reported as an option for unruptured cerebral aneurysm clipping. ${ }^{1-4}$ The main goal is to minimize the risk of stroke or related neurological deficit during either temporary or permanent aneurysm clipping by performing immediate intra-operative neurological assessment. ${ }^{3}$ Although there are theoretical advantages of performing this surgery in awake conditions, the challenges and potential complications also need to be considered. That's why it is not widely practiced in the world, and not many anesthetists are fond of it. Our report highlights the anesthesia concerns, management and challenges during our first successful anesthetic management of this surgery.

\section{Case Report}

A 49-year-old man with an underlying history of bronchial asthma and chronic smoking presented to the emergency department with worsening right-sided headache, associated with nausea and vomiting, and right eye blurring and double vision for a week before 
admission. The symptoms initially preceded by a history of dizziness and blurring of vision for the past two months. Initial assessment in the emergency department was uneventful, and he achieved the highest Glasgow Coma Scale (GCS) score. He was discharged with simple analgesics.

He came back a few days later with worsening of headache and ptosis. On assessment, the GCS score was the highest, blood pressure (BP) was 150/80 $\mathrm{mmHg}$, heart rate (HR) was 73 beats/min and oxygen saturation $\left(\mathrm{SpO}_{2}\right)$ was $100 \%$. Pupils were bilaterally reactive and $2 \mathrm{~mm}$ in size on the left side and $3 \mathrm{~mm}$ in size on the right. Cranial nerve examination revealed right 3rd, 5th, and 8th nerve abnormalities, clinically shown by right eye ptosis, reduced sensation over the right trigeminal nerve distribution and right sensorineural hearing loss. Other neurological examinations were unremarkable. The provisional diagnosis by the neuro-medical team was multiple cranial neuropathies secondary to either compressive lesions from abnormal vasculature or space-occupying lesions, and the differential diagnosis was polyneuritis cranialis. Magnetic resonance imaging of the brain revealed an aneurysm in the right internal carotid artery and middle cerebral artery (ICA-MCA) bifurcation. Computed tomography angiography (CTA) with three-dimensional reconstruction confirmed the diagnosis of a $1.7 \mathrm{~mm}$ diameter saccular aneurysm at the junction of the right ICA and anterior choroidal artery. The patient was subsequently planned for a right pterional craniotomy and aneurysm clipping using the awake craniotomy technique, in view of the risk of accidental clipping of the anterior choroidal artery with the aneurysm, which might lead to ipsilateral internal capsule infarction and dense hemiplegia.

During the preoperative assessment, the awake craniotomy option, the technique, risks and benefits were explained to the patient. The awake technique was planned under a combination of scalp block and monitored anesthesia care using dexmedetomidine, target controlled infusion (TCI) remifentanil as well as TCI propofol. In the operating theater, standard monitoring was applied, including noninvasive $\mathrm{BP}$, electrocardiography and $\mathrm{SpO}_{2}$. Oxygen at $31 / \mathrm{min}$ was delivered via a nasal prong with an end-tidal carbon dioxide $\left(\mathrm{EtCO}_{2}\right)$ monitoring. Intraarterial cannulation for invasive BP monitoring and a triple-lumen central venous catheter was inserted under local anesthesia. A cerebral state monitor (CSM) was also applied for depth of sedation monitoring. Two 18G intravenous (IV) cannulas were inserted for fluids and drug administration. One of the IV cannulas was dedicated to TCI remifentanil and TCI propofol. A laryngeal mask airway and an endotracheal tube were also prepared in case emergency airway management was required during surgery.The aims of the anesthesia technique were to keep deep sedation during scalp block, skull head-pinning, surgical incision, craniotomy, and earlier surgical exploration. After that, the patient was planned to be kept awake for neurological assessment during temporary and permanent aneurysm clipping. The deep sedation phase was to restart during the surgical closure period. Dexmedetomidine was planned as the main sedative agent, backed up with TCI propofol if needed. The analgesia was achieved mainly with scalp block using a $30 \mathrm{ml}$ mixture of $0.75 \%$ ropivacaine with $1: 200,000$ adrenaline and TCI remifentanil.

Deep sedation was started with $1 \mu \mathrm{g} / \mathrm{kg}$ loading dose of dexmedetomidine over $10 \mathrm{~min}$ and followed by a maintenance dose of $0.7 \mu \mathrm{g} / \mathrm{kg} / \mathrm{hr}$. TCI remifentanil was also started at a target effect concentration of 0.5 $1.0 \mathrm{ng} / \mathrm{ml}$, aiming at a CSM score between 60 and 70 for deep sedation. The scalp block was infiltrated bilaterally at 12 sites of scalp innervations. ${ }^{5}$ The bladder was catheterized for urine output monitoring after the scalp block. An additional $5 \mathrm{ml}$ lignocaine 2\% was infiltrated at the pinning sites before head positioning using head-skull pinning. After successful pinning, the patient was positioned supine with the head slightly flexed and rotated $30^{\circ}$ to the left. A sandbag was placed under the ipsilateral shoulder to minimize stretching of the brachial plexus. IV ceftriaxone $1.5 \mathrm{~g}$ was administered as a prophylactic antibiotic and IV dexamethasone $8 \mathrm{mg}$ and IV ondansetron $4 \mathrm{mg}$ were administered as prophylactic anti-emetics. The patient's face was adequately exposed to ensure the patient's comfort and allow direct observation of breathing and facilitating communication with the patient during assessment. During the pterional craniotomy, dexmedetomidine infusion was titrated between $0.5-0.7 \mu \mathrm{g} / \mathrm{kg} / \mathrm{hour}$, TCI remifentanil between $1.0-1.5 \mathrm{ng} / \mathrm{ml}$ and additional TCI propofol between $0.5-1.0 \mu \mathrm{g} / \mathrm{ml}$, according to the surgical stimulation, patient response and CSM score 
of 60-70. There was an episode of severe bradycardia down to 30 beats/min during a high dose of dexmedetomidine infusion, which was successfully treated with an IV atropine $0.5 \mathrm{mg}$ bolus. The patient also developed snoring during the deep sedative state; however, there was no episode of desaturation. Before the dura opening, the patient developed cough and wheezing, possibly due to oral secretions. However, it also raised the suspicion of venous air embolism. The surgeon was informed and the surgical field was flooded with saline and bone wax was applied to the bone edges. The patient was otherwise stable hemodynamically and supplementary oxygen was given via face mask with regular oral cavity suctioning.

Approximately $2.5 \mathrm{~h}$ into the surgery, while the neurosurgeon was approaching the aneurysm, the sedation was withheld and the patient was kept awake. At this point, noradrenaline infusion had been readied for induced hypertension management after permanent clipping. After brief temporary artery clipping, the aneurysm was managed to be clipped. The patient was continuously assessed for neurological function during the clipping by the second neurosurgeon. The patient initially responded very well during the assessment, but after the application of permanent clipping he did not respond and the motor assessment showed hemiplegia. Immediate induced hypertension was initiated by titration of a noradrenaline infusion up to $10 \mu \mathrm{g} / \mathrm{kg} / \mathrm{min}$, aiming for a systolic BP of 160-180 $\mathrm{mmHg}$. After a few minutes of achieving a systolic BP of $160 \mathrm{mmHg}$, the patient responded, and motor function was recovered. The noradrenaline infusion was continued, and the patient was subsequently sedated again for surgical closure. Acetaminophen $1 \mathrm{~g}$ was infused for post-operative analgesia. After

\section{Table 1: Anaesthesia concerns for awakecraniotomy and aneurysm clipping}

\begin{tabular}{|c|c|}
\hline Anesthesia concerns for awake craniotomy & Anesthesia concerns for aneurysm clipping \\
\hline $\begin{array}{l}\text { - Patient cooperation during neurological } \\
\text { assessment }\end{array}$ & - Risk of intraoperative aneurysm rupture \\
\hline $\begin{array}{l}\text { - Fixed head positioning intraoperatively } \\
\text { which leads to difficulty in airway } \\
\text { management }\end{array}$ & $\begin{array}{l}\text { - Risk of ischemia leading to hemiplegia } \\
\text { and neurological deterioration during } \\
\text { temporary clipping }\end{array}$ \\
\hline $\begin{array}{l}\text { - Patient comfort during long surgery in } \\
\text { fixed surgical position }\end{array}$ & $\begin{array}{l}\text { - Risk of neurological deterioration during } \\
\text { permanent clipping }\end{array}$ \\
\hline - Risk of venous air embolism & $\begin{array}{l}\text { - Hemodynamic control throughout } \\
\text { procedure }\end{array}$ \\
\hline - Risk of intraoperative seizure & - Long duration of surgery \\
\hline
\end{tabular}

surgery, the patient was monitored in the postanesthesia care unit. He was still mildly sedated but able to obey commands and move all four limbs. He was then transferred to the neurosurgical intensive care unit (Neuro-ICU) for close observation.

In the Neuro-ICU, pain was controlled with acetaminophen $1 \mathrm{~g}$ 8-hourly and fentanyl infusion of $10 \mu \mathrm{g} / \mathrm{h}$. The highest pain score was $5 / 10$ in the immediate post-operative period and subsequently reduced to less than 3/10. Fentanyl infusion was stopped $24 \mathrm{~h}$ after surgery. The GCS score was the highest throughout, and the patient did not exhibit any signs of cerebral vasospasm or bleeding. Daily transcranial Doppler scans also showed no evidence of cerebral vasospasm. The patient was discharged home without any neurological deficit.

\section{Discussion}

Awake craniotomy for clipping of unruptured cerebral aneurysms has not been widely practiced, but interest has been resurged after it was recently described in a few reports. A report by Luders et al. in 2005 described three cases of awake testing during distal vessel occlusion for mycotic aneurysm. ${ }^{1}$ In the same year, Chen et al. described awake visual testing for clipping of ophthalmic artery aneurysms. ${ }^{4}$ In 2013, Passacantilli et al. described awake testing during the trapping of a giant fusiform MCA aneurysm, ${ }^{2}$ and Suzuki et al. reported in the following year four cases of awake testing during clipping of anterior choroidal artery aneurysms. ${ }^{6}$ Thirty cases of awake craniotomy for unruptured aneurysm clipping were reported by Abdulrauf et al. in 2017. ${ }^{3}$ In 2018, we reported our center's first case of a successful procedure. ${ }^{7}$ However, these case reports mainly focused on surgical issues without much discussion on anesthesia management.

Anesthesia
concerns for this
surgery include
anesthesia for
awake craniotomy
and of aneurysm
clipping, as


summarized in Table 1. The anesthesia objectives for this surgery are to keep the patient awake for neurological assessment during temporary or permanent clipping; to maintain hemodynamic stability throughout surgery; to provide good analgesia during surgery and to prevent intraoperative complications, such as aneurysm rupture, cerebral edema or stroke. A case series of 30 cases of awake unruptured cerebral aneurysm surgery concluded that this technique had the potential advantage of detecting early neurological deterioration and decreasing the risk of ischemic injury, based on the findings from three patients who developed asynchronous clinical neurological and neurophysiological changes. ${ }^{3}$

Previous reports on the awake craniotomy technique were all for unruptured aneurysms. ${ }^{1-4}$ It is possible that in future cerebral aneurysm with subarachnoid hemorrhage (SAH) patients with Hunt and Hess class I-II or World Federation of Neurosurgical (WFNS) SAH scale of I-II can also be considered, if the patient has recovered from the headache symptoms and can cooperate and communicate intraoperatively.

Based on our experience, awake craniotomy for clipping of aneurysms is more challenging than awake craniotomy for other indications. The most challenging part is preventing intraoperative rupture of the aneurysm by controlling hemodynamic parameters and preventing neurological deficit during temporary and permanent artery clipping. Two anesthesia techniques have been discussed for awake craniotomy for other indications, either the asleep-awake-asleep technique using a laryngeal mask airway or a monitored anesthesia care technique with scalp block. ${ }^{8}$ We believe that monitored anesthesia care (MAC), in combination with scalp block, is a better technique for awake cerebral aneurysm surgery. Repeated airway manipulation during the asleep-awake-asleep technique might stimulate a sympathetic surge and sudden changes in the transmural pressure of the aneurysm, which might lead to rupture of the aneurysm. In our patient, a combination of dexmedetomidine, TCI remifentanil, TCI propofol and scalp block were critical in providing adequate analgesia and controlling hemodynamic parameters. In our center, all awake craniotomy procedures have mainly been performed under a MAC technique. ${ }^{5} \mathrm{~A}$ report by Abdulrauf et al. also described the use of dexmedetomidine $\quad(0.2-1.0 \mu \mathrm{g} / \mathrm{kg}$ per hour), remifentanil $(0.05-2.00 \mu \mathrm{g} / \mathrm{kg} / \mathrm{min})$ and scalp block with $0.5 \%$ ropivacaine as their technique. ${ }^{3}$

We used a TCI technique for both propofol and remifentanil because the infusion was easily titratable with the TCI technique. The use of TCI of propofol and remifentanil during awake craniotomy has been described in a previous report. ${ }^{9}$ We used TCI propofol as a backup drug to dexmedetomidine to deepen the sedation as well as control the blood pressure. Nitroglycerin and esmolol have also been used in treating blood pressure during awake aneurysm surgery. ${ }^{10}$ Most earlier reported cases on awake cerebral aneurysm surgery were performed under the asleep-awake-asleep anesthesia technique..$^{2,4,6}$ In our practice, invasive monitoring, including a central venous line (CVL) for central venous pressure monitoring, intra-arterial BP monitoring and central bladder drainage (CBD) for urine output monitoring were all required. BP control is important during cerebral aneurysm surgery, and therefore intra-arterial cannulation for BP monitoring was performed under local anesthesia before starting the sedation. CVL is critical for vasopressor or inotropic support after permanent clipping or in a situation where they need to be started if the hemodynamic parameters are unstable because the aneurysm ruptured. CBD is important for urine output monitoring in view of the risk of rupture and prolonged surgery. Other indications of awake craniotomy, such as for tumor excision or deep brain stimulation surgery, might not need CVL, intra-arterial BP and CBD. We also used CSM to guide our depth of sedation level instead of bispectral index monitoring, because its electrode was smaller and less space was required to occupy the forehead area.

One of the main goals of this surgical technique in the awake state is immediate clinical assessment during and after clipping, so that immediate management can be performed to prevent permanent neurological deficit. All sedation was stopped during neurological assessment. If after temporary or permanent clipping the patient develops hemiplegia, the surgeon can either release or reapply clipping, while reassessing the functions. In this study, the surgeon was satisfied with the clip placement, but the patient did not respond to the assessment and showed signs of hemiplegia immediately after clipping. In this situation, the role of anesthesia team was critical to induce hypertension by 
starting vasopressors so that BP could be augmented and collateral blood flow improved for reperfusion of the ischemic area and potentially improving neurological function. Our patient recovered neurological function after induced hypertension up to a systolic BP of $150-160 \mathrm{mmHg}$. Another intraoperative incident for our patient was sudden symptoms of cough and wheezing before the dura opening, which was possibly due to oral secretions but also raised the suspicion of venous air embolism (VAE). Appropriate management actions were taken to anticipate the possibility of VAE. The risk of VAE is one of the known complications during awake craniotomy, and cough symptoms are one of its earlier symptoms. ${ }^{11}$

Post-operative analgesia was managed with acetaminophen, fentanyl infusions and parecoxib 40 mg every $12 \mathrm{~h}$ for two days with patient-controlled analgesia (PCA). Fentanyl as a backup can also be an option. ${ }^{5}$ The use of scalp block might also help to reduce post-operative pain. Hwang et al. showed that the use of scalp block with $0.75 \%$ levobupivacaine improved recovery profiles, effectively lowered postoperative pain and PCA consumption and also reduced post-operative antihypertensive agent as well as incidence of post-operative nausea vomiting in patients who underwent elective fronto-parietal craniotomy for aneurysm clipping. ${ }^{12}$ The advantages of the awake procedure of unruptured cerebral aneurysm clipping are lower neurological morbidity, lower mortality and shorter hospital stay. ${ }^{10}$ In the recent case series, the median length of stay was 3 days and the median modified Rankin scale score was $1 .^{3}$ The use of the awake technique might also reduce the need for expensive neuromonitoring during the procedure, such as electroencephalography, somatosensory or motor evoked potentials, which can give rise to false-positive or false-negative results during the procedure. Clinical examination and assessment are still the best assessment for neurological functions.

\section{Conclusion}

In conclusion, awake craniotomy for unruptured cerebral aneurysm clipping is challenging. Good patient selection, proper anesthesia planning and anticipation of potential complications are critical for the success of anesthesia and surgical management.

\section{Acknowledgment}

I acknowledge the Director of Hospital Universiti Sains Malaysia for supporting the services of neuroanesthesia and neurosurgery.

\section{Conflicts of interest}

None declared by the authors.

\section{Author contributions}

WMNWH: Concept, manuscript writing \& editing

SCO: Concept, manuscript writing

WTF: Manuscript writing

ZI, RK: Concept, manuscript editing

\section{References}

1. Luders JC, Steinmetz MP, Mayberg MR. Awake craniotomy for microsurgical obliteration of mycotic aneurysms: technical report of three cases. Neurosurgery 2005;56:E201; discussion E201.

2. Passacantilli E, Anichini G, Cannizzaro D, Fusco F, Pedace $F$, Lenzi J, et al. Awake craniotomy for trapping a giant fusiform aneurysm of the middle cerebral artery. Surg Neurol Int 2013;4:39.

3. Abdulrauf SI, Vuong P, Patel R, Sampath R, Ashour AM, Germany L.M, et al. "Awake" clipping of cerebral aneurysms: report of initial series. I Neurosurg 2017;127:311-318.

4. Chen P, Dunn IF, Aglio LS, Day AL, Frerichs KU, Friedlander RM. Intraoperative awakening for vision examination during opthalmic artery aneurysm clipping: technical case report. Neurosurgery 2005;66:E440.

5. Mohd Nazaruddin WH, Mohd Fahmi L, Laila AM, Zamzuri I, Abdul Rahman IZ, Hardy MZ. Awake Craniotomy: A Case Series of Anaesthetic Management using a Combination of Scalp Block, Dexmedetomidine and Remifentanil in Hospital Universiti Sains Malaysia. Med J Malaysia 2013;68:64-66.

6. Suzuki K, Mikami T, Sugino T, Wanibuchi M, Miyamoto $\mathrm{S}$, Hashimoto N, et al. Discrepancy between voluntary movement and motor-evoked potentials in evaluation of motor function during clipping of anterior circulation aneurysms. World Neurosurg 2014;82:e739-745.

7. Idris Z, Kandasamy R, Neoh YY, Abdullah JM, Wan Hassan WMN, Mat Hassan ME. The First Awake Clipping of a Brain Aneurysm in Malaysia and in ASEAN: Achieving International Standards. Malays J Med Sci 2018;25:1-4.

8. Eseonu Cl, ReFaey K, Garcia O, John A, QuinonesHinojosa A, Tripathi P. Awake Craniotomy Anesthesia: A Comparison of the Monitored Anesthesia Care and Asleep-Awake-Asleep Techniques. World Neurosurg 2017:104:679-686. 
9. Hans $\mathrm{P}$, Bonhomme V, Born JD, Maertens de Noordhoudt A, Brichant JF, Dewandre PY. Targetcontrolled infusion of propofol and remifentanil combined with bispectral index monitoring for awake craniotomy. Anaesthesia 2000;55:255-259.

10. Abdulrauf SI. Awake craniotomies for aneurysms, arteriovenous malformations, skull base tumors, high flow bypass, and brain stem lesions. J Craniovertebr Junction Spine 2015;6:8-9.
11. Wan Hassan WMN, Yeap BT, Ab Mukmin L, Ghani ARI, Mohd Zaini RH. An unusual case of repeated venous air embolism during awake bilateral deep brain stimulation surgery. Southern African Journal of Anaesthesia and Analgesia 2016;22:42-44.

12. Hwang JY, Bang JS, Oh CW, Joo JD, Park SJ, Do SH, et al. Effect of scalp blocks with levobupivacaine on recovery profiles after craniotomy for aneurysm clipping: a randomized, double-blind, and controlled study. World Neurosurg 2015;83:108-113. 